\title{
Research Article \\ Four-Terminal Square Piezoresistive Sensors for MEMS Pressure Sensing
}

\author{
Awlad Hossain ${ }^{1}$ and Ahsan Mian ${ }^{2}$ \\ ${ }^{1}$ Department of Engineering and Design, Eastern Washington University, Cheney, WA 99004, USA \\ ${ }^{2}$ Department of Mechanical and Materials Engineering, Wright State University, Dayton, OH 45435, USA \\ Correspondence should be addressed to Ahsan Mian; ahsan.mian@wright.edu
}

Received 28 September 2016; Revised 11 December 2016; Accepted 18 December 2016; Published 11 January 2017

Academic Editor: Fanli Meng

Copyright (C) 2017 A. Hossain and A. Mian. This is an open access article distributed under the Creative Commons Attribution License, which permits unrestricted use, distribution, and reproduction in any medium, provided the original work is properly cited.

\begin{abstract}
The sensitivity of four-terminal piezoresistive sensors commonly referred to as van der Pauw (VDP) structure is investigated. The VDP sensor is considered to be fabricated on (100) silicon due to its potential application in MEMS (microelectromechanical systems) pressure sensors. The sensitivity of the VDP sensor may be affected by misalignment during the etching/diffusion process, the nonuniformity of piezoresistive coefficients through the sensor thickness, and pad size with respect to the sensor size. For this particular analysis, the effect of VDP stress sensitivity on variations in pad sizes and through-the-thickness $\pi$-coefficient variation are studied as the effect of misalignment has already been investigated by researchers. Two three-dimensional (3D) finite element analysis (FEA) models are first developed for both traditional VDP resistance and equivalent four-wire bridge measurements. Then, the FEA models are validated with the closed form analytical solutions for point contacts ("zero" pad size) under different biaxial loads. Once the FEA models are validated, additional simulations are conducted to understand the influence of different parameters on the voltage measurements for an equivalent four-wire bridge configuration. It is observed that pad size and throughthe-thickness nonuniformity in piezoresistive constants adversely affect the sensor sensitivity.
\end{abstract}

\section{Introduction}

Piezoresistive materials offer a change in electrical "resistivity" when a mechanical stress or pressure is applied. Although most or all materials exhibit some degree of piezoresistivity, researchers are interested in far fewer materials. The most common of these materials is single crystal "silicon," although polycrystalline silicon also exhibits the effect [1]. Piezoresistive materials usually act as a transducer and generate an electrical signal as a function of applied stress. Pressure sensing is a fundamental measurement in many engineering applications. In the automotive industry, for example, a typical passenger car has pressure sensors as part of the powertrain, brake, airbag, and tire pressure monitoring systems $[2,3]$. In the biomedical industry, pressure sensors find application in invasive and noninvasive monitoring of blood intracranial and epidural pressure [4]. Pressure sensors can also be used to indirectly measure other variables such as fluid/gas flow, speed, water level, and altitude. Across other industries, pressure sensors are ubiquitous in testing and control. For many years, varieties of pressure sensing devices such as capacitive, resonance, optical, and strain gage pressure sensors have been used. Capacitive sensors typically have one fixed and one flexible conductive plate, which flexes when subjected to pressure. The capacitance between the plates changes as a function of pressure. The resonance pressure sensors exploit a vibrating element, often a cantilever or cylinder. The air pressure changes the resonance properties of the structure due to structural deformation, and the shift in resonant frequency is measured electrically or optically and correlated to pressure. The optical pressure sensors use laser to measure nanometer scale deflection of a diaphragm surface that is displaced under pressure. Similarly, the piezoresistive strain gage sensors measure strain on the surface of a pressure diaphragm but use a diaphragm of piezoresistive material and exploit the piezoresistive effect of the gage to achieve pressure.

In this research, we have used four-terminal piezoresistive pressure sensing structures as shown in Figure 1. A traditional 


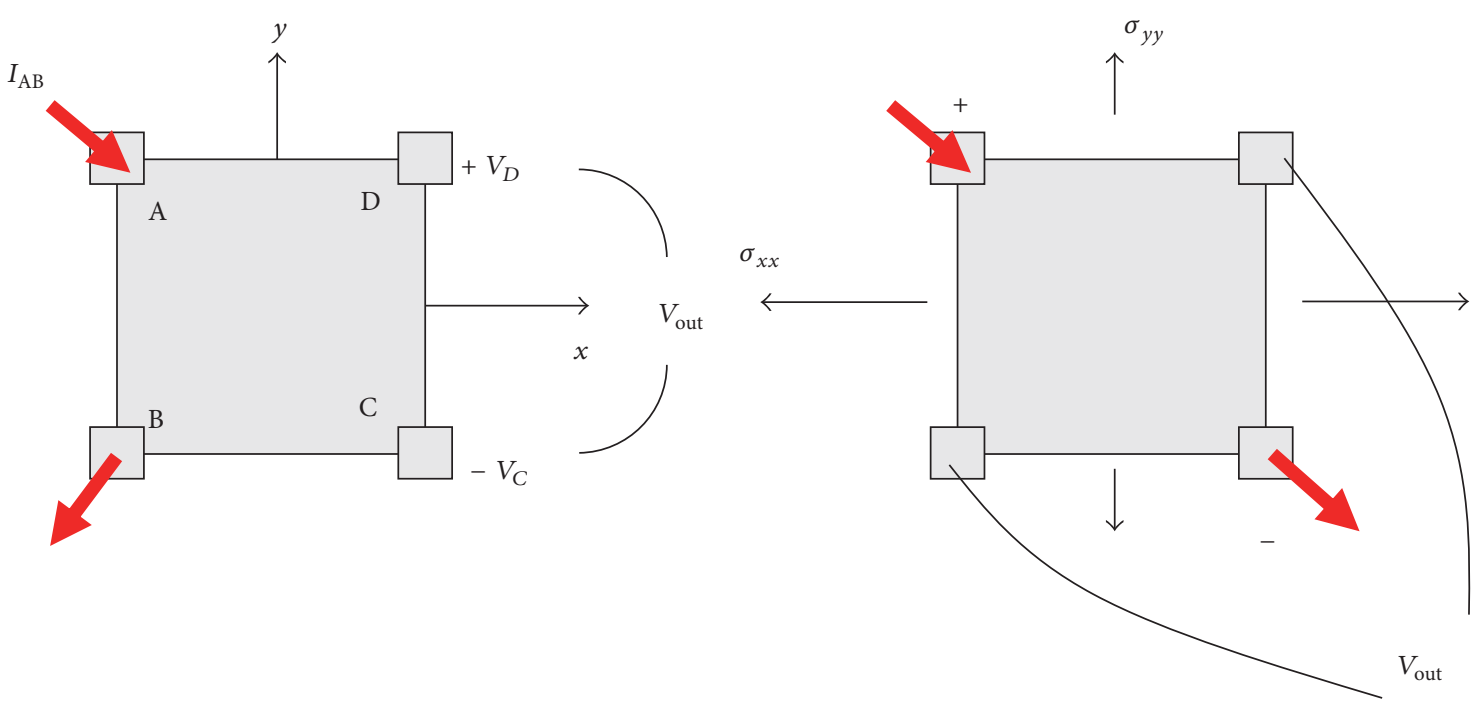

(a) The traditional van der Pauw (VDP) sensor measurement

(b) Diagonal sensor measurement

Figure 1: Measurement schemes for four-terminal sensors.

four-terminal conductor commonly called van der Pauw (VDP) sensor is shown in Figure 1(a). The VDP sensor is a four-terminal piezoresistive stress sensing structure based on the sheet resistance measurement technique developed by van der Pauw $[5,6]$. The VDP structures could be used as pressure sensors and have potentiality to avoid limitations of conventional pressure sensors as discussed earlier. In fact, the VDP structures are found to have higher stress sensitivity compared to the conventional sensors for determining stress or pressure $[7,8]$. The VDP sensor is usually made of piezoresistive material such as silicon, and its resistance can be measured from knowing the current $\left(I_{\mathrm{AB}}\right)$ and voltage $\left(V_{\mathrm{CD}}\right)$. The current is usually supplied at terminals (pads) $A$ and $B$, and voltage is measured at terminals (pads) $C$ and D. Finally, the resistance $(R)$ or change in resistance $(\Delta R)$ is calculated from $V_{\mathrm{CD}} / I_{\mathrm{AB}}$. It was shown in $[9,10]$ that the normalized resistance change requires two separate measurements with current supplied individually at adjacent sides. Jaeger et al. [9] presented numerical and experimental results for four-wire bridge-mode operation (Figure 1(b)) that eliminates two separate resistance measurements required for a traditional square VDP sensor. It is shown that the output voltage from the four-wire bridge measurements is directly related to the traditional VDP resistance measurements. For equivalent four-wire bridge measurements, voltage is applied to the devices across one diagonal, and the output voltage is measured across the other diagonal, thereby producing a single four-wire measurement that is directly proportional to either in-plane shear stress or normal stress difference depending upon the overall sensor orientation $\left(0^{\circ}\right.$ or $45^{\circ}$ with the wafer flat direction). It is worth mentioning that the single diagonal current loading measurement is more convenient and less time-consuming compared to the two separate adjacent current loading measurements as mentioned in [7, 8]. Therefore, the diagonal voltage loading is particularly more suitable for IC implementations.
This research aims to characterize the sensitivity of the VDP structures with changing different factors related to VDP fabrication and measurements. In particular, this paper investigates the sensitivity of the VDP sensor by studying its piezoresistive response with changing the pad size. The piezoresistivity of Si material is normally induced by doping, where the impurities are added to the surface of the base material. Therefore, the piezoresistivity might not be the same or uniform throughout the thickness of the sensor. In this paper, the effect of nonuniform $\pi$-coefficients resulting from nonuniform dopant concentration on sensor response is investigated. We also demonstrated the equivalency between the standard VDP measurement and an equivalent four-wire bridge measurement (diagonal technique) for the completeness of this paper. The paper is organized as follows. Section 2 briefly represents the theoretical background of stress dependence piezoresistive VDP sensors with showing electrical symmetries inherent in the structure. It also includes the superposition analysis to establish the expected response in voltage for two adjacent loadings compared to single diagonal loading. Section 3 represents the finite element modeling with necessary boundary conditions and loading. The numerical results are shown in Section 4, which are compared with theoretical calculations.

\section{Theoretical Background}

Figure 2 represents the schematic diagram of (100) silicon wafer with a VDP structure on it. The unprimed $\left(x_{1}-x_{2}\right)$ coordinate system is aligned with silicon crystallographic axes, whereas the axes of the primed coordinate system $\left(x_{1}^{\prime}-x_{2}^{\prime}\right)$ are aligned with the wafer flat direction. For this study, the VDP sensor is considered to be oriented along the in-plane primed coordinates and denoted by $x$-y-axes. The silicon resistor is assumed to have uniform thickness $(t)$, piezoresistivity $(\rho)$, and four contact points, labeled A 


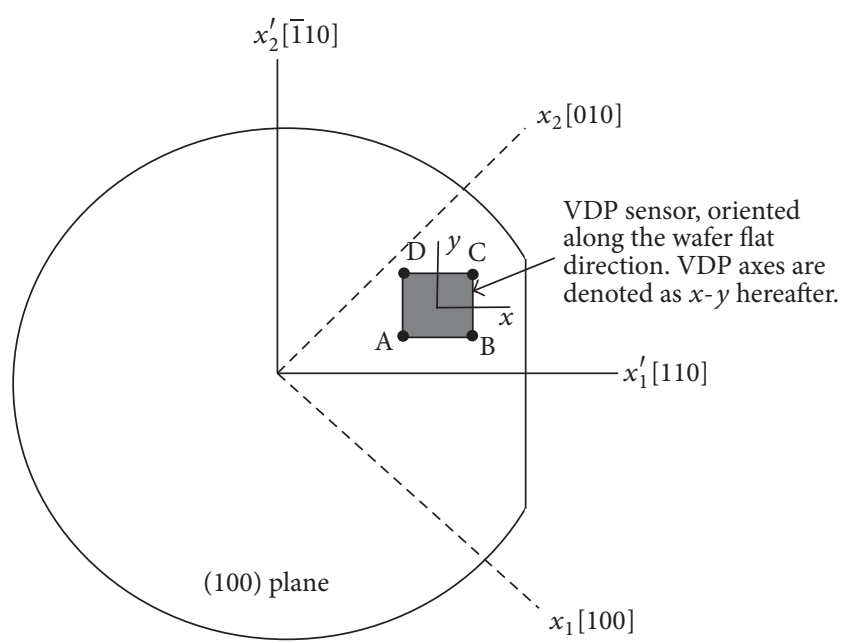

Figure 2: (100) silicon wafer and an oriented rectangular VDP sensor.

to D, with no isolated holes. Such a conductor is electrically isotropic in the unstressed condition but becomes anisotropic when subjected to stress.

The original theory was developed first by van der Pauw $[5,6]$ to calculate the "change in resistance" for a given piezoresistive material. For a planar isotropic piezoresistive conductor of arbitrary shape with no discontinuity, VDP theorem defines the "resistance" $R_{\mathrm{AB}, \mathrm{CD}}$ as the potential difference $\left(V_{\mathrm{D}}-V_{\mathrm{C}}\right)$ between contacts $\mathrm{D}$ and $\mathrm{C}$ per unit current through contacts $\mathrm{A}$ and $\mathrm{B}, I_{\mathrm{AB}}$ (see (1)). In this case, current enters the sample through contact $\mathrm{A}$ and leaves through contact B. A simplified notation is introduced for representing the resistance of the oriented VDP sensor as follows:

$$
R_{0}=R_{\mathrm{AB}, \mathrm{CD}}=\frac{V_{\mathrm{D}}-V_{\mathrm{C}}}{I_{\mathrm{AB}}} .
$$

A similar resistance $R_{\mathrm{BC}, \mathrm{DA}}$ which is equivalent to $R_{90}$ is defined in an analogous manner. For a rotationally symmetric structure, such as the proposed square VDP structure, the unstressed resistances $R_{\mathrm{AB}, \mathrm{CD}}$ and $R_{\mathrm{BC}, \mathrm{DA}}$ are equal. The unstressed resistance for a square sensor, according to the original VDP theorem $[5,6]$, can also be expressed as

$$
R_{\mathrm{AB}, \mathrm{CD}}=R_{\mathrm{BC}, \mathrm{DA}}=\frac{\rho \ln 2}{\pi t}=R^{0,0} .
$$

Here, $\rho$ is the resistivity and $t$ is the thickness of the conductor. Superscripts in $R^{0,0}$ represent zero stress and zero temperature variation from room or reference temperature. The unstressed resistance will be used to normalize the resistance change, called "Normalized Resistance Change Difference (NRCD)," for a stressed VDP conductor using the following equation:

$$
\mathrm{NRCD}=\frac{\Delta R_{0}}{R_{0}}-\frac{\Delta R_{90^{\circ}}}{R_{90^{\circ}}}=\frac{R_{0}^{\sigma}-R_{0}^{0}}{R_{0}^{0}}-\frac{R_{90^{\circ}}^{\sigma}-R_{90^{\circ}}^{0}}{R_{90^{\circ}}^{0}} .
$$

Subscript represents the orientation of the sensor with respect to primed axis as shown in Figure 2. Figure 3 shows the scheme of $R_{0}$ and $R_{90}$ measurements. The superscripts $\sigma$ and 0 , used in (3), represent the stressed and unstressed states used in calculating the resistance. Please note that $\Delta R / R$ in (3) is referred to as normalized resistance change (NRC).

In this paper, the electrical behavior of the VDP device is explored for two different configurations. First, the current is injected at two "adjacent" corners, and voltage is determined against the other two "adjacent" corners for 0- and 90-degree orientations. This configuration, which is already shown in Figure 3, was applied by Mian et al. [7, 8]. Second, the current is injected at two "diagonal" corners, and voltage is determined against the other two "diagonal" corners. This configuration is shown in Figure 4(c). It is shown in detail in [9] that the combination of two adjacent loadings as shown in Figures 4(a) and 4(b) is equivalent to a single diagonal loading as shown in Figure 4(c). A brief description is provided here for the completeness of the paper. Using superposition theory in Figure 4,

$$
\begin{aligned}
V_{o} & =\left(V_{\mathrm{VDP} 1}+V_{1}\right)+\left(-V_{\mathrm{VDP} 2}-V_{2}\right) \\
& =\left(V_{\mathrm{VDP} 1}-V_{\mathrm{VDP} 2}\right)+\left(V_{1}-V_{2}\right) .
\end{aligned}
$$

Based upon electrical symmetry for the unstressed isotropic situation, $V_{\mathrm{VDP} 1}=V_{\mathrm{VDP} 2}=V_{\mathrm{VDP}}$ and $V_{1}=V_{2}$. Here, $V_{\mathrm{VDP}}$ is the VDP voltage given by van der Pauw $[5,6]$ for any rotationally symmetric structure, which is reexpressed as

$$
V_{\mathrm{VDP}}=I R_{\mathrm{VDP}}=I \frac{\rho \ln 2}{\pi t} .
$$

When stress is present, the VDP sensor becomes anisotropic and $[5,6]$ have shown theoretically that the combination of the two separate sets of measurements in Figures 4(a) and 4(b) results as follows for the 0-degree VDP device on (100) silicon:

$$
V_{\mathrm{VDP} 1}-V_{\mathrm{VDP} 2}=3.16 * V_{\mathrm{VDP}}\left[\pi_{44}\left(\sigma_{11}^{\prime}-\sigma_{22}^{\prime}\right)\right]
$$

This output voltage difference provides 3.16-fold improvement in sensitivity over the corresponding resistor rosettes and bridges. Therefore, the transverse output voltage for diagonal loading becomes

$$
V_{0}=3.16 * V_{\mathrm{VDP}} *\left[\pi_{44}\left(\sigma_{11}^{\prime}-\sigma_{22}^{\prime}\right)\right]+\left(V_{1}-V_{2}\right) .
$$

Note that $V_{1}=V_{2}$ by symmetry in the unstressed case. Therefore, the second term involving $V_{1}$ and $V_{2}$ is zero. Finite element simulations and experimental results in [9] also demonstrated that $V_{1}$ and $V_{2}$ still cancel out even in stressed sensors. Subsequently, (7) for diagonal loading becomes

$$
V_{0}=3.16 * V_{\mathrm{VDP}} *\left[\pi_{44}\left(\sigma_{11}^{\prime}-\sigma_{22}^{\prime}\right)\right]
$$

In this paper, the finite element simulation for diagonal loading is compared with (8), along with (5). 


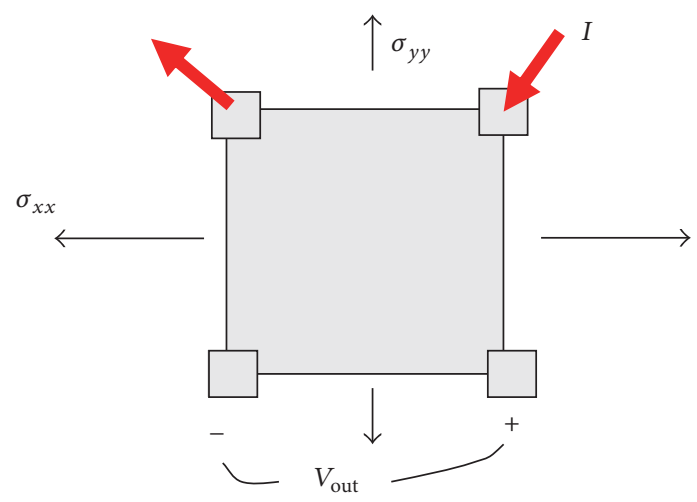

$R_{0}$ measurement

(a)

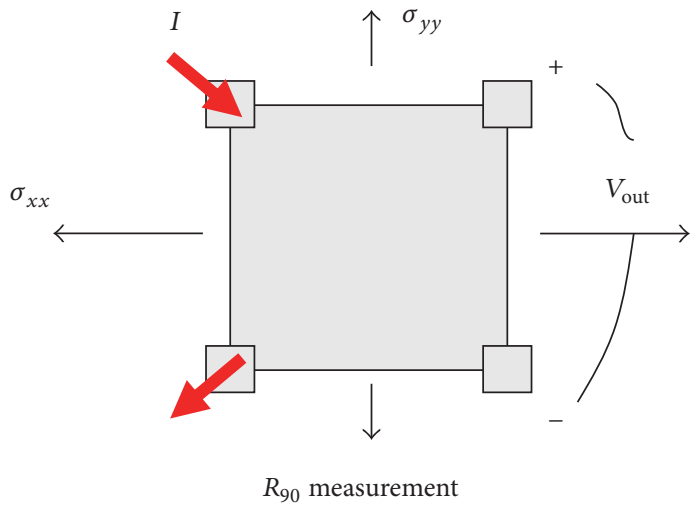

(b)

FIGURE 3: Measurement scheme for $R_{0}$ and $R_{90}$ in calculating the NRCD.

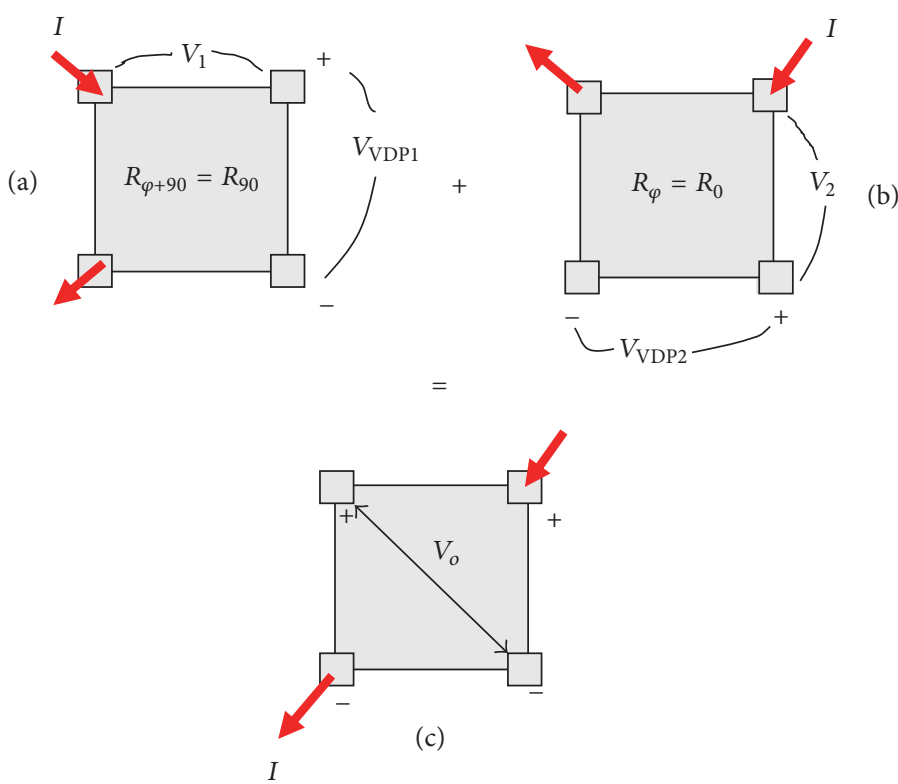

FIGURE 4: VDP configurations. Adjacent loadings as shown in (a) and (b). Equivalent diagonal loading as shown in (c).

\section{Finite Element (FE) Model}

The finite element analysis (FEA) method has been adopted to predict the piezoresistive response of the VDP sensor. A 3D FEA model, representing both VDP sensor and substrate, is developed first using commercially available FEA software ANSYS. In particular, we have used APDL-ANSYS Parametric Design Language. The piezoresistive analysis has been conducted utilizing the appropriate load and boundary conditions that a VDP sensor experiences. The numerical prediction is then compared with analytical results to validate the FEA modeling scheme. Once the FEA model is validated, additional analyses are conducted for sensitivity analysis as mentioned above.

MEMS pressure sensors typically consist of a square diaphragm having sensors placed at a location of maximum stress developed due to applied pressure. At this location, the sensor is typically subjected to biaxial stress [10], and hence the biaxial stress sensitivity of the sensor should be investigated. The VDP model consists of a small region of material in which the doped VDP structure is embedded. Figure 5 represents the finite element model used for the VDP sensor and underlying substrate. The substrate is modeled with 3D 20-node structural solid element (SOLID186) that exhibits quadratic displacement behavior. The element is defined by 20 nodes having three degrees of freedom per node: translations in the nodal $x, y$, and $z$ directions. The VDP sensor is modeled with 3D 20-node coupled-field solid element (SOLID226) with "piezoresistive" capability. The element is defined by 20 nodes having four degrees of freedom per node: translations in the nodal $x, y$, and $z$ directions and voltage. It is worth mentioning here that ANSYS Element Library does not include any 8-node "piezoresistive" element. However, it offers flexibility to drop the midsize 

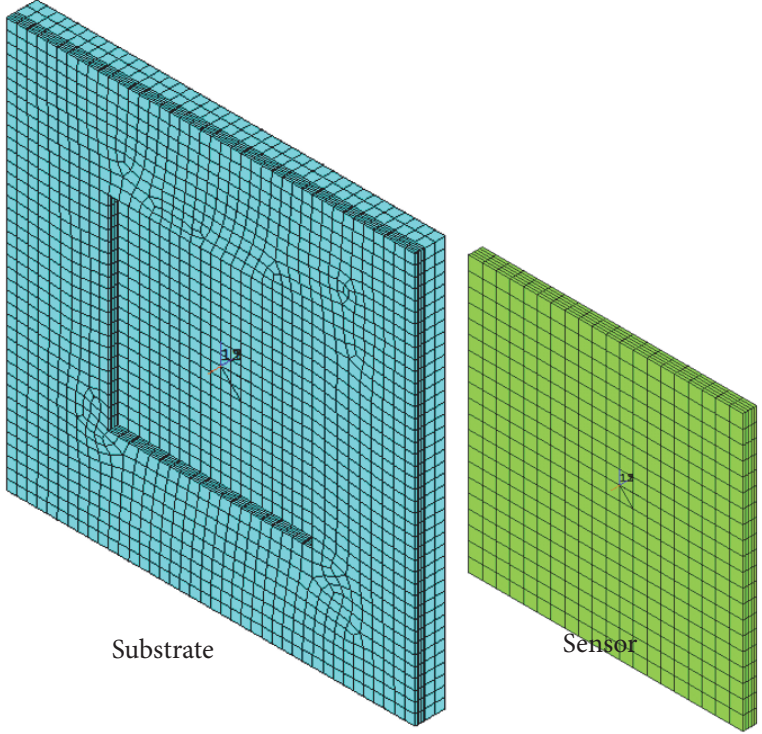

FIGURE 5: Finite element model used for the VDP sensor and the substrate.

TABLE 1: Anisotropic and piezoresistive coefficients used in simulation.

\begin{tabular}{lccc}
\hline \multicolumn{2}{c}{ Anisotropic } & \multicolumn{2}{c}{ Piezoresistive } \\
Coefficient & $\mathrm{MPa}$ & Coefficient & $\mathrm{Pa}^{-1}$ \\
\hline$C_{11}$ & $16.57 \times 10^{4}$ & $\pi_{11}$ & $66 \times 10^{-12}$ \\
$C_{12}$ & $6.39 \times 10^{4}$ & $\pi_{12}$ & $-11 \times 10^{-12}$ \\
$C_{44}$ & $7.96 \times 10^{4}$ & $\pi_{44}$ & $1380 \times 10^{-12}$ \\
\hline
\end{tabular}

nodes if required. The typical element shape associated with SOLID186 and SOLID226 is available in ANSYS Element Library [11].

Both the substrate and the sensor are modeled with "anisotropic" material properties. The anisotropic elasticity matrix is generated by providing the required elasticity data in a tabular form. In this case, three different sets of elasticity data, labeled by $C_{11}, C_{12}$, and $C_{44}$, are provided. These data are shown in Table 1. However, the elements associated with the VDP sensor have piezoresistive capabilities to calculate the change in electrical resistivity produced by elastic stress or strain. Material properties, associated with sensor elements, include the electrical resistivity and the piezoresistive matrix besides the elastic coefficient matrix. In this simulation, the electrical resistivity of p-type $\mathrm{Si}$ material was considered to be $7.8 \times 10^{-8}$ teraohms. Values of the piezoresistive coefficients, commonly called $\pi$-coefficients $\left(\pi_{11}, \pi_{12}\right.$, and $\left.\pi_{44}\right)$, in lightly doped p-type Si materials that are used in this simulation are also shown in Table 1.

The side dimensions of the VDP sensor and the underlying substrate were $100 \times 100$ and $200 \times 200$ microns, respectively. The thickness of the sensor and the substrate was 5 microns and 15 microns, respectively. The doped VDP sensor was embedded (incorporated) within the substrate as shown in Figure 5. Finer meshing was used for the sensor and associated substrate regions, followed by coarse meshing

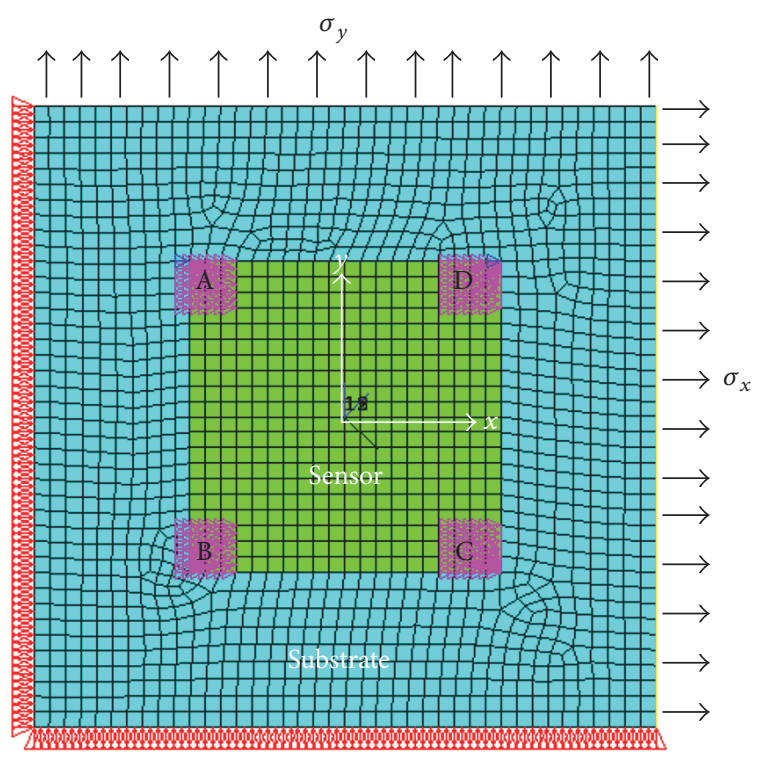

FIgURE 6: Boundary conditions and loading assigned to FE model.

towards the substrate back surface, as shown in Figure 5. The following boundary conditions were implemented in FE model, as shown in Figure 6. The symmetric boundary condition was assigned to the substrate, attached with a roller at the left and bottom sides. This condition implies that the left side of the substrate was constrained to move along global $x$ direction $\left(u_{x}=0\right)$, where it was allowed to move along global $y$ direction. Similarly, the bottom side of the substrate was constrained to move along global $y$ direction $\left(u_{y}=0\right)$, where it was allowed to move along global $x$ direction. Pressure was applied as a surface force to the right side of the substrate. The electrical boundary condition (voltage) was assigned to the sensor. In particular, the voltage DOF was specified at the upper left (pad A) and upper right (pad D) corners as $+10 \mathrm{~V}$ and $+0 \mathrm{~V}$, respectively. Additionally, the output voltage or voltage difference was determined from corners located at the lower left (pad B) and lower right (pad C) to approximate the connection that would physically occur if this device were connected by vias.

\section{Results and Discussion}

The FEA model was solved for two different configurations, which could be called "adjacent" and "diagonal" loadings for "point" contacts or "zero" pad size. The adjacent loading scenario is shown in Figures 4(a) and 4(b), for which we have determined the NRCD as shown in (3). The diagonal loading scenario is shown in Figure 4(c), for which we have determined the transverse voltage, which could also be analytically solved by using (5) and (8). For both cases, the model was solved first in an unstressed state and then subsequently solved for different stressed states.

In the stressed state of adjacent loading, the normalized resistance was calculated for 0 -degree and 90-degree sensor orientations. The "simulated resistance," as shown in (1), was calculated from the "voltage difference over current." 


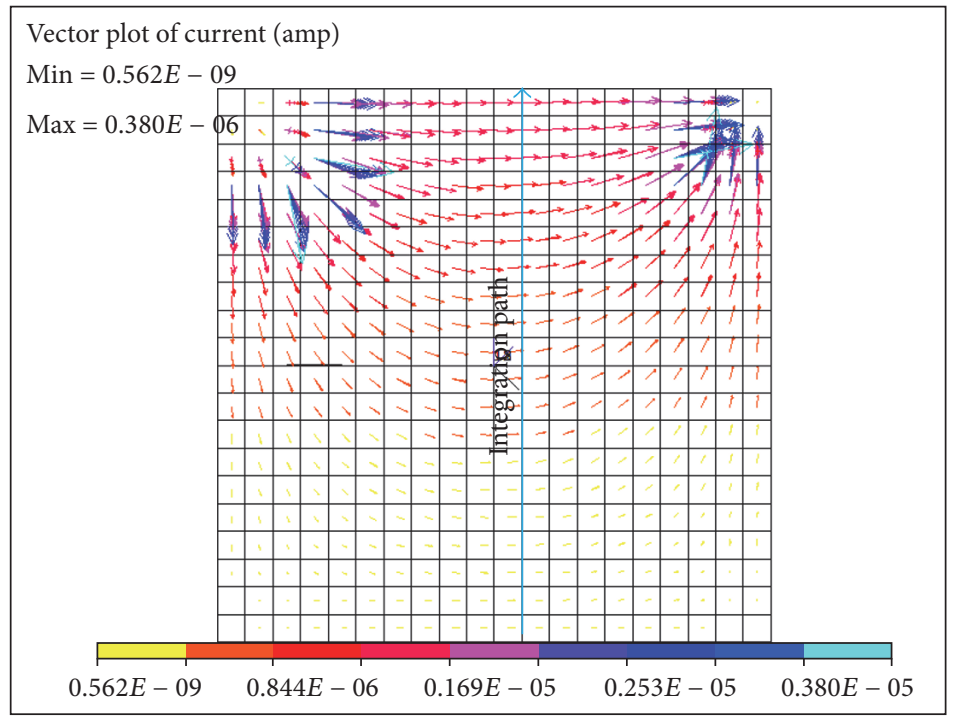

(a)

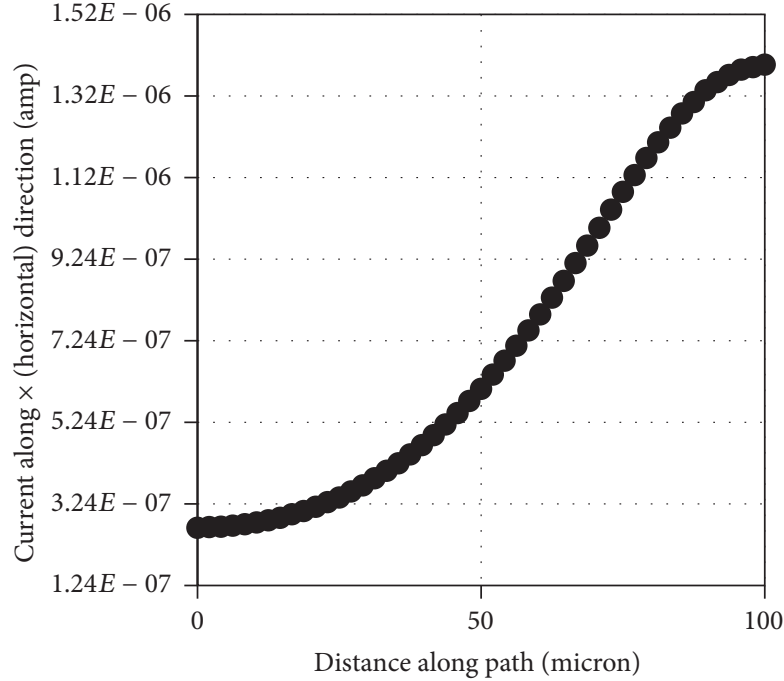

(b)

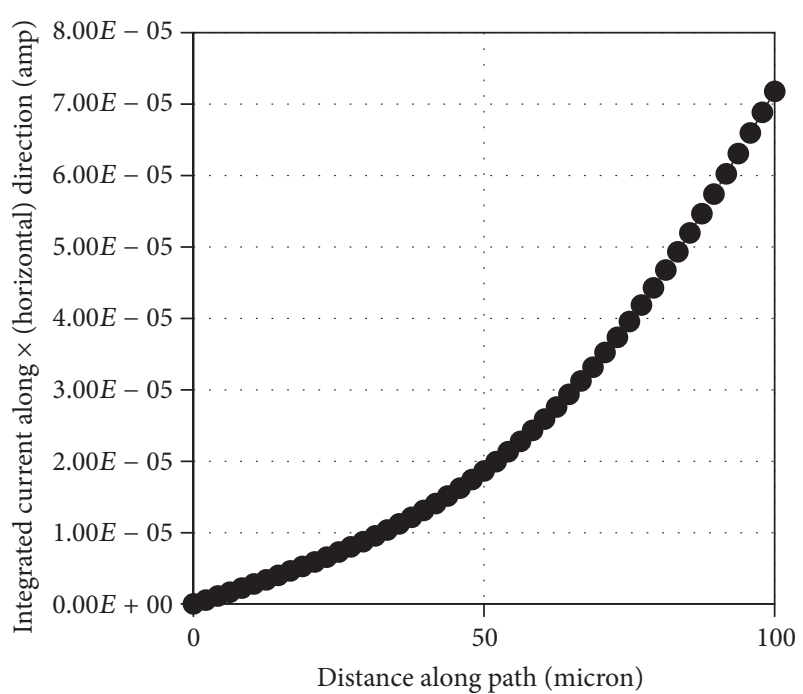

(c)

FIGURE 7: (a) Current vector of the VDP sensor for 0-degree orientation. (b) Current density and (c) integrated current along global $x$ direction mapped onto a path, as shown in (a).

The voltage difference and current were obtained as output parameters. The voltage difference was determined from the output voltage of pads B and C. The current could be determined by two different ways. It could be determined from a current vector mapping onto a path created along the sensor width direction. Then, the current was integrated along the path length. Finally, the total current was determined from the integrated current multiplied by the sensor thickness. Besides this method, the current could also be accurately determined as a reaction solution (force) from the nodes which were grounded (i.e., voltage $=0$ ). For example, Figure 7(a) represents the current vector of the VDP sensor for an unstressed sensor for $R_{0}$ resistance. Current starts from pad A and exits through pad D, as expected. Figures 7 (b) and 7(c) represent the current and integrated current, respectively, along the global $x$ direction mapped onto a path shown in Figure 7(a). In case of diagonal loading, an electrical voltage was applied at two diagonal corners (e.g., at pads A and C). A typical contour plot of applied voltage is shown in Figure 8(a). The current vector and the current flow along a diagonal path are shown in Figures 8(b) and 8(c), respectively. All numerical results were found to be intuitively correct. The current flows from pad $\mathrm{A}$ to pad $\mathrm{C}$, and the maximum current flow occurred at the middle of the path, as expected.

For "adjacent" loading, the "normalized resistance change (NRC)" was calculated from both FEA and theory for "point" conductors. First, the resistance of the VDP sensor was obtained as a function of increasing biaxial stress $\left(\sigma_{x}-\right.$ $\left.\sigma_{y}\right)$ applied to the substrate, as shown in Figure 6. To calculate $\operatorname{NRC}\left(\Delta R_{0}\right)$ or $\operatorname{NRC}\left(\Delta R_{90}\right)$, stressed and unstressed 


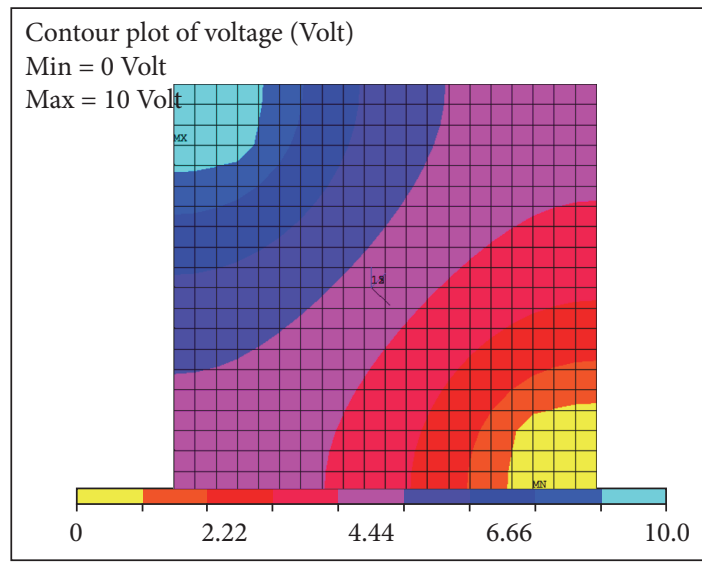

(a)

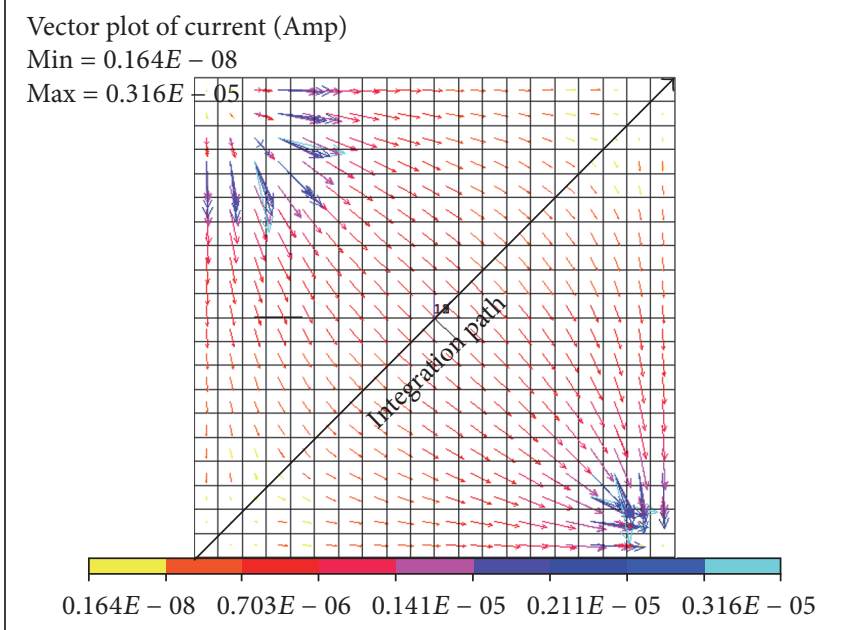

(b)

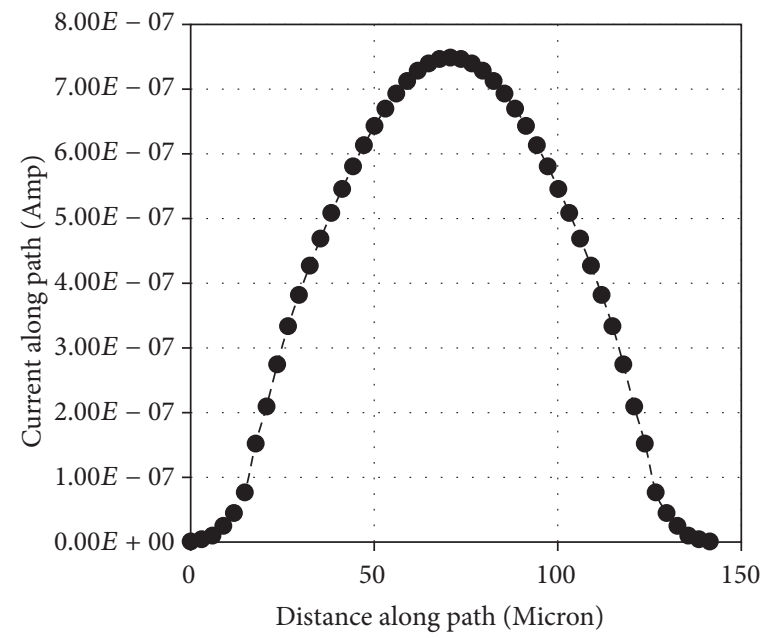

(c)

FIGURE 8: (a) Contour plot of applied voltage for diagonal loading. Current flows from the upper left corner and gets out through the lower right corner. (b) The current density vector for diagonal loading. (c) Current flow along a path for diagonal loading.

resistances were obtained by dividing the output voltage with the current (according to (1)) from the FE simulations. The NRC was also calculated analytically using the equations used in [8]. The results of the NRC calculation are shown in Figure 9. The numerical results were found to be in good agreement with theoretical solutions for both 0 - and 90degree sensors. The NRC, at both orientations, was found to start from zero and increased with increasing the biaxial stresses. The individual slope at each orientation agreed well with the theory presented in [8].

Numerical simulations were then conducted for several diagonal loadings to demonstrate that the transverse (diagonal) voltage is equivalent to the two sequential adjacent voltage measurements, as shown in Table 2 along with Figure 10. The data represents the numerical results comparing the difference in the two VDP adjacent voltages with the voltage difference across the diagonal for p-type sensor on (100) silicon. Numerical results for diagonal loading were also compared with theoretical voltage computed by (5) and (8). This comparison is shown in Figure 11, where excellent matching was observed between the FEA and the theoretical results.

The above discussions establish the fact that, for point conductors, the diagonal measurements are equivalent to the NRCD measurements for typical VDP sensors. Thus, the diagonal measurements can provide same advantages (e.g., temperature independence and 3.16 times the sensor response over resistor sensors) with a single measurement. Therefore, the following discussions are restricted to the diagonal measurements only.

Additional simulations were performed to investigate the effects of pad size and nonuniformity in piezoresistive constants, as shown in Figures 12(a) and 14(a), respectively. The "current" flow through the source conductors was monitored for different pad sizes. It was observed that the current flow remains almost the same with stresses for any given pad size; 
TABLE 2: Comparison between transverse voltage and VDP adjacent voltages.

\begin{tabular}{lcccc}
\hline Stress $(\mathrm{MPa})$ & $V_{0}$ & $V_{90}$ & $V_{0}-V_{90}$ & $V_{\text {Diag }}$ or $V_{\text {out }}$ \\
\hline 0 & 0.11569 & 0.11569 & 0.00000 & 0.00000 \\
10 & 0.11809 & 0.11335 & 0.00474 & 0.004736 \\
20 & 0.12052 & 0.11105 & 0.00947 & 0.009473 \\
30 & 0.12318 & 0.10828 & 0.01490 & 0.01495 \\
40 & 0.12600 & 0.10606 & 0.01994 & 0.019943 \\
50 & 0.12867 & 0.10375 & 0.02492 & 0.024930 \\
60 & 0.13138 & 0.10146 & 0.02992 & 0.029920 \\
70 & 0.13412 & 0.09921 & 0.03491 & 0.034911 \\
\hline
\end{tabular}

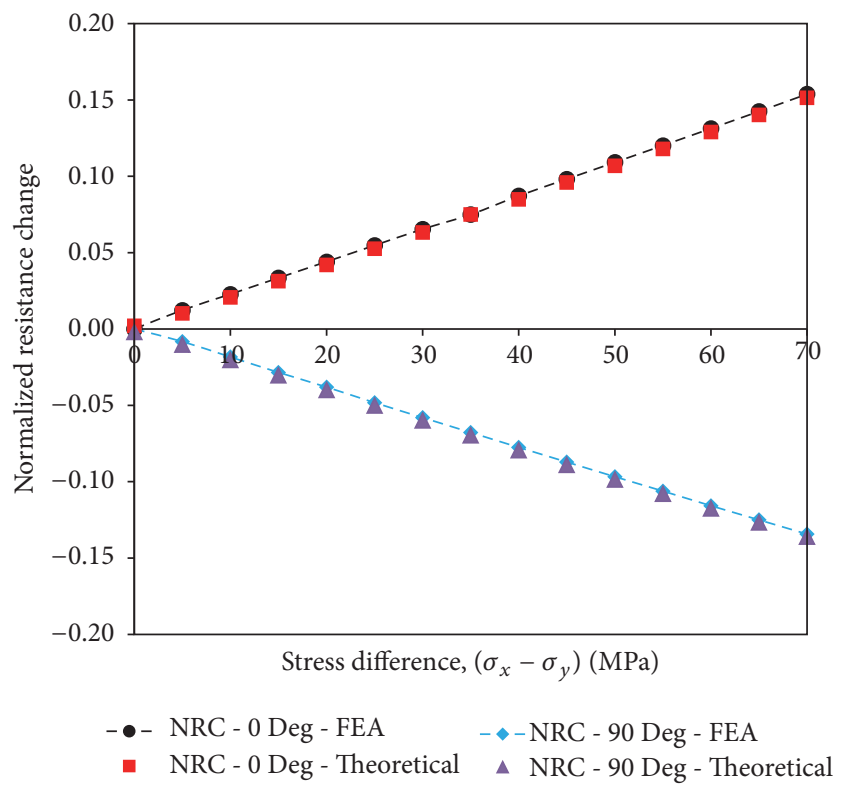

FIGURE 9: Normalized resistance change with stress difference.

however, it increases with increasing the pad sizes, as shown in Figure 12(b). This result is intuitively correct because the increased pad area will result in increased current for any given source voltage.

The effects of pad size on output voltage are shown in Figure 13. It is observed from Figure 13(a) that the slope of the voltage response curve (a.k.a. "gage factor") increases with increased pad sizes. This result does imply that the sensor sensitivity increases with the increase in pad size. To further understand the influence of pad size on sensor response, the output voltage per unit current flow is plotted against normal stress difference and is shown in Figure 13(b). It is clear that the larger the pad size, the lower the sensor sensitivity. For example, $15 \mu \mathrm{m}$ pad ( $15 \%$ of sensor dimension) causes a $13 \%$ reduction in sensitivity when compared with the point contact sensors. Note that it is not feasible to design sensors with point contacts; hence, this reduction in sensor sensitivity cannot be avoided. Even with this reduced sensitivity, the four-contact VDP sensor will still be superior when compared with conventional serpentine resistor sensors because it has already been demonstrated that the VDP sensors have 3.16 times higher sensitivity $[8,9]$.

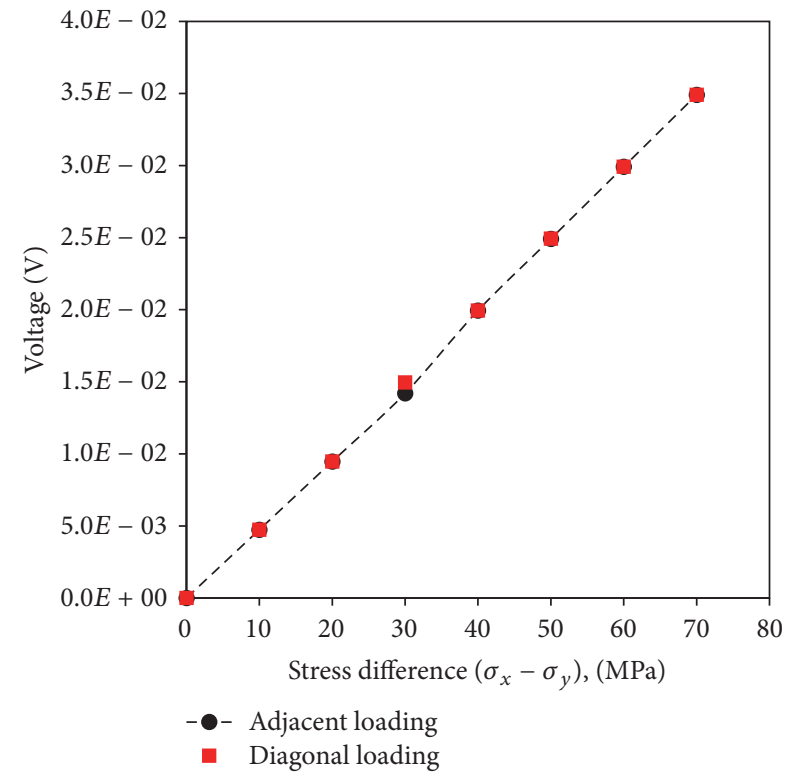

FIGURE 10: Comparison between transverse voltage and VDP adjacent voltages.

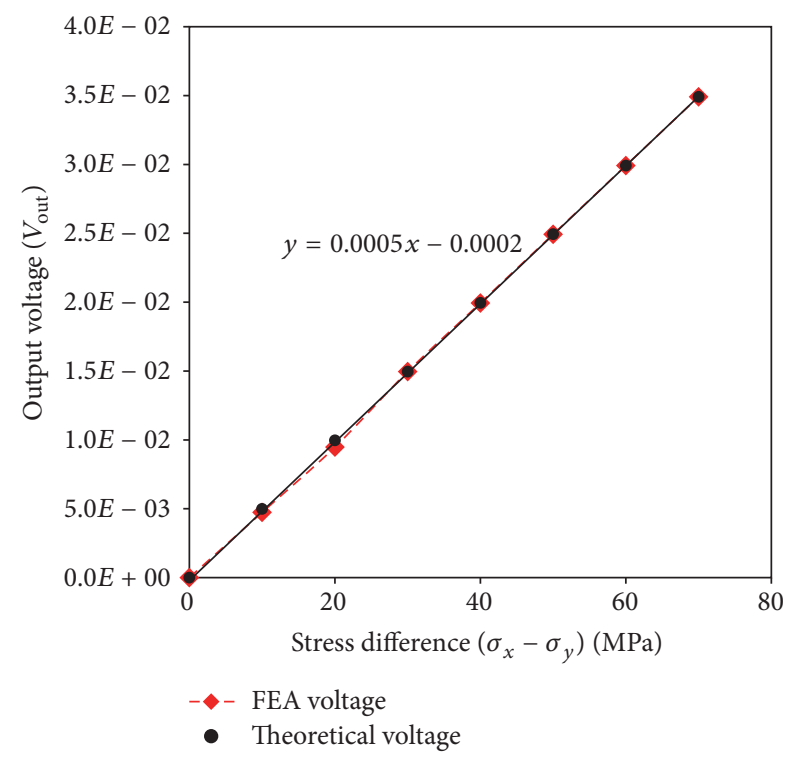

FIGURE 11: Comparison between FEA and theoretical voltage for diagonal loading for supply voltage of $10 \mathrm{~V}$. 


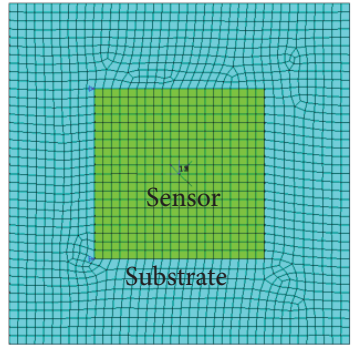

Point Pad

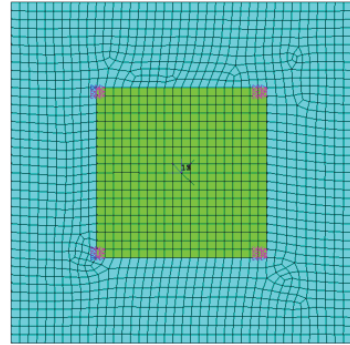

Square pad: $5 \times 5$

sq. microns

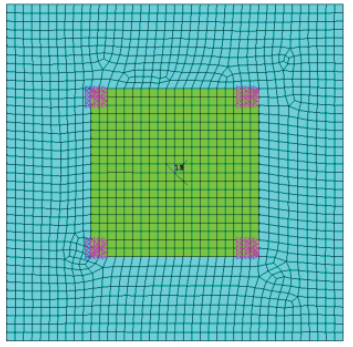

Square pad: $10 \times 10$

sq. microns

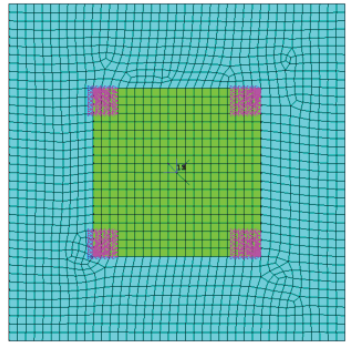

Square pad: $15 \times 15$

sq. microns

(a)

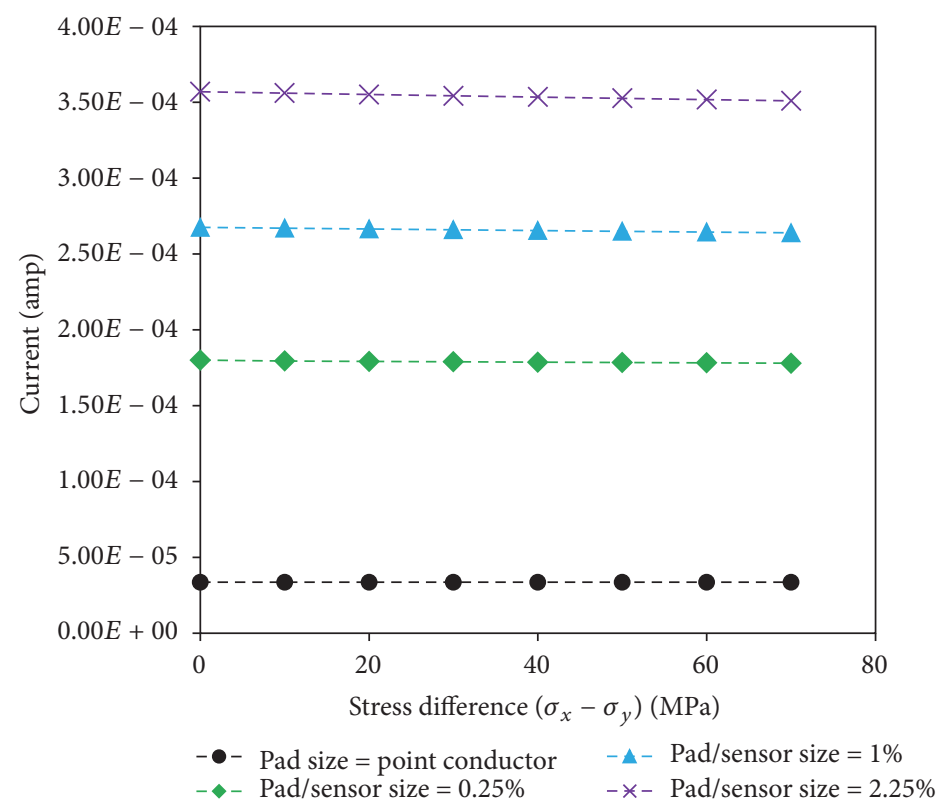

(b)

FIGURE 12: (a) FE models showing VDP sensors with different pad sizes. (b) FE results showing total current flow through the supply conductors.

Next, the effect of nonuniform $\pi$-coefficients resulting from nonuniform dopant concentration on sensor response is investigated. As mentioned before, the piezoresistivity might not be the same or uniform throughout the thickness of the sensor. Numerical simulations were conducted to investigate the effects of nonuniform resistivity and $\pi$-coefficients on NRCD. In this case, the sensor was modeled into 4 different layers along the thickness direction, as shown in Figure 14(a). Each layer of sensor was also modeled with 3 rows of elements. "Layer \# 1" represents the "topmost layer," which was considered to have $100 \%$ resistivity and $\pi$-coefficients, whose values are listed in Table 1. The subsequent layers were assumed to have reduced resistivity and $\pi$-coefficients. For example, simulations were conducted considering "Layer \# 2," "Layer \# 3," and "Layer \# 4" to have $75 \%, 50 \%$, and $25 \%$ resistivity and $\pi$-coefficients, respectively, compared to "Layer \# 1." The numerical result of NRCD for the point conductor with nonuniform resistivity and $\pi$-coefficients is shown in Figure 14(b). It is observed that the sensor sensitivity goes down substantially with nonuniform $\pi$ coefficients compared to uniform properties. It is also found to be reduced further with nonuniform resistivity and $\pi$ coefficients.

\section{Conclusions}

In this research, the sensitivity of a VDP structure under the variations of pad size for different biaxial stress states is investigated. First, 3D finite element models were developed representing piezoresistive four-contact VDP sensors fabricated on silicon diaphragm. Adjacent and diagonal loadings were investigated and demonstrated that the diagonal output voltage measurement is equivalent to the two sequential adjacent voltage (equivalent to NRCD) measurements. In other words, a four-contact square sensor requiring one-diagonal output voltage measurements replaces the traditional VDP measurements and still possesses 3.16-fold enhanced sensitivity over traditional serpentine resistor sensors. 


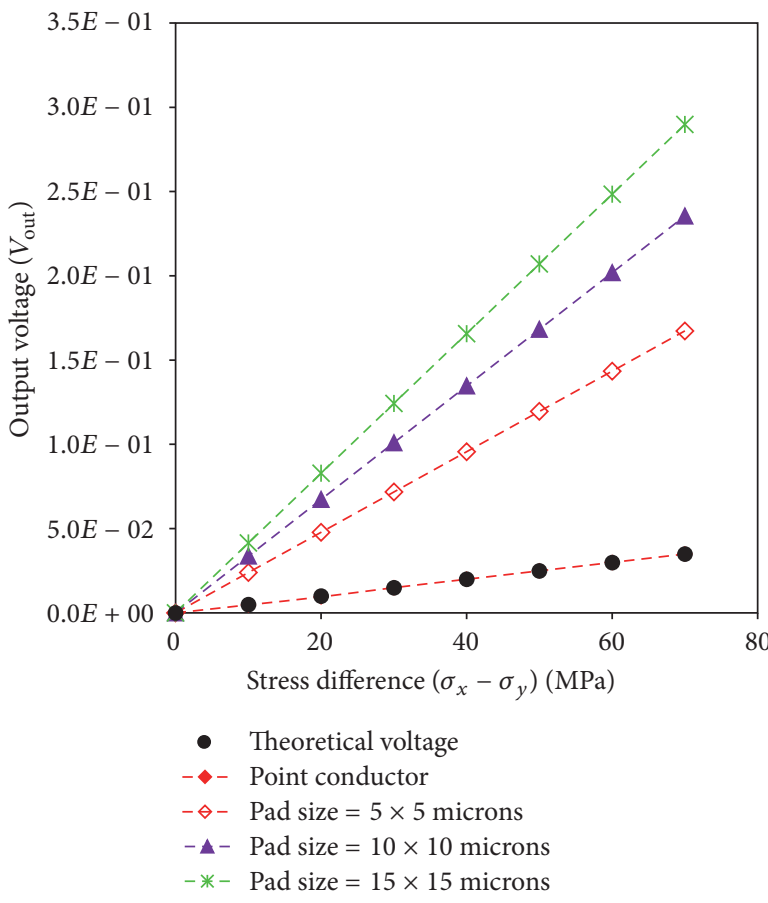

(a)

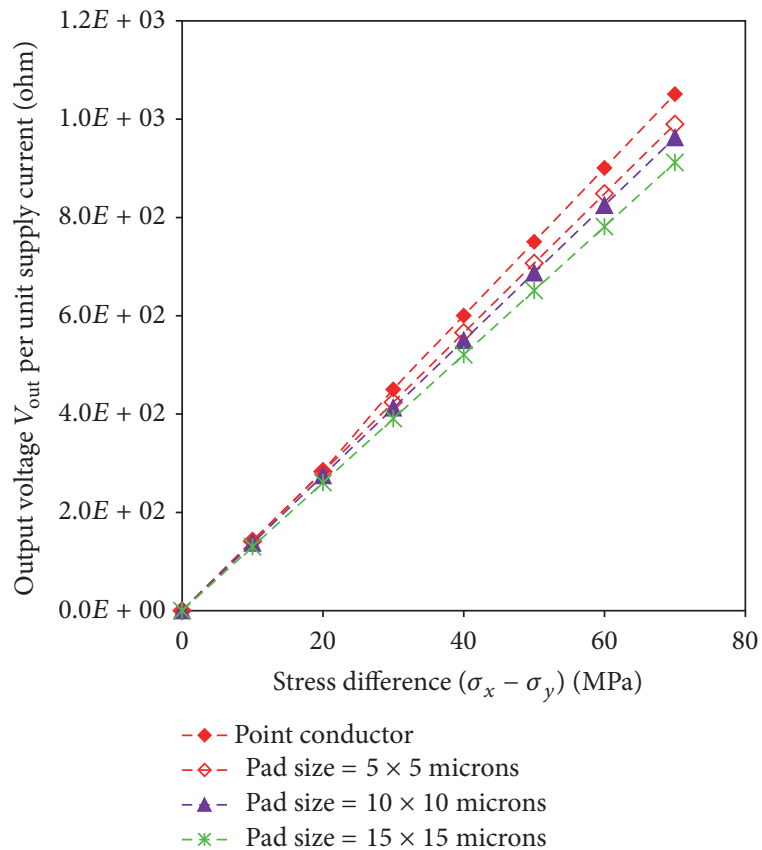

(b)

Figure 13: (a) The effect of pad size on output voltage (diagonal loading). (b) The effect of pad size on transverse resistance (diagonal loading).
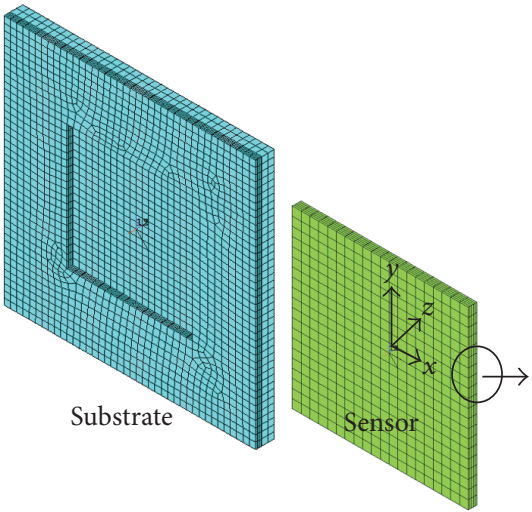

(a)

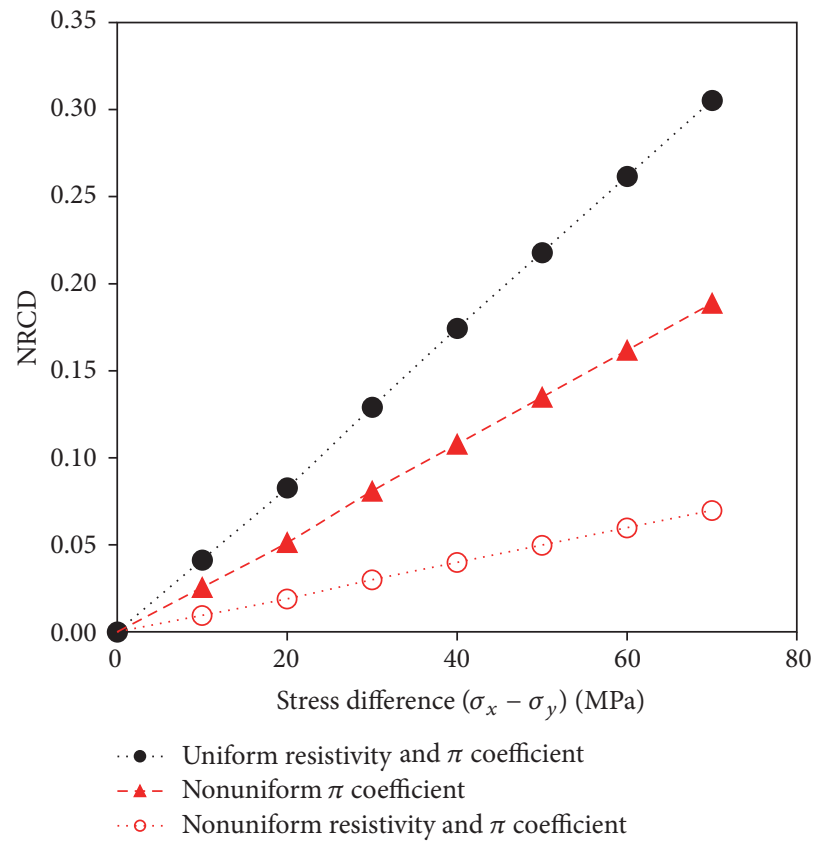

(b)

FIGURE 14: (a) Pictorial expression for different layers of sensor to assign nonuniform resistivity and $\pi$-coefficients. (b) The simulated NRCD with nonuniform resistivity and $\pi$-coefficients assigned to different layers of sensor.

Next, the voltage output $\left(V_{\text {out }}\right)$ of the sensor was calculated to predict its sensitivity for different pad sizes. $V_{\text {out }}$ was observed to vary linearly with in-plane normal stress difference $\left(\sigma_{x}-\sigma_{y}\right)$ for applied pressure. The FEA predicted $V_{\text {out }}$ matches very well the theoretical values for point contact sensors. It is observed that the sensor sensitivity decreases with increasing pad size.

Additional numerical simulations were conducted to investigate the effects of nonuniform resistivity and $\pi$ coefficients along the thickness direction of the sensor. The 
simulated sensor sensitivity was found to be reduced for the nonuniform properties compared to the uniform properties. It is also concluded that the numerical scheme adopted here is reasonably accurate and hence can be used for detailed design of VDP based MEMS pressure sensor.

\section{Competing Interests}

The authors declare that there are no competing interests regarding the publication of this paper.

\section{References}

[1] P. J. French and A. G. R. Evans, "Polycrystalline silicon as a strain gauge material," Journal of Physics E: Scientific Instruments, vol. 19, no. 12, pp. 1055-1058, 1986.

[2] R. Schmidt, "Universal intelligent pressure transducer for automotive applications," Elektronic Praxis, no. 20, pp. 62-64, 2000.

[3] J. P. Schuster, "Automotive silicon based pressure transducers for fluid power applications," in Proceedings of the National Conference on Fluid Power, Annual Meeting, 1986.

[4] S. Marco, J. Samitier, O. Ruiz, J. R. Morante, and J. Esteve, "Highperformance piezoresistive pressure sensors for biomedical applications using very thin structured membranes," Measurement Science and Technology, vol. 7, no. 9, pp. 1195-1203, 1996.

[5] L. J. van der Pauw, "A method of measuring specific resistivity and Hall effect of discs of arbitrary shape," Philips Research Reports, vol. 13, no. 1, pp. 1-9, 1958.

[6] L. J. van der Pauw, "Determination of resistivity tensor and hall tensor of anisotropic conductors," Philips Research Reports, vol. 16, pp. 187-195, 1961.

[7] A. Mian, J. C. Suhling, and R. C. Jaeger, "Sensitivity of (100) silicon VDP sensors to uniaxial and hydrostatic loads," in Proceedings of the 20th Southeastern Conference on Theoretical and Applied Mechanics (SECTAM-XX '00), Callaway Gardens, Ga, USA, April 2000.

[8] A. Mian, J. C. Suhling, and R. C. Jaeger, "The van der Pauw stress sensor," IEEE Sensors Journal, vol. 6, no. 2, pp. 340-356, 2006.

[9] R. C. Jaeger, M. Motalab, S. Hussain, and J. C. Suhling, "Fourwire bridge measurements of silicon van der Pauw stress sensors," Journal of Electronic Packaging, Transactions of the ASME, vol. 136, no. 4, Article ID 041014, 10 pages, 2014.

[10] A. Mian and J. Law, "Geometric optimization of van der Pauw structure based MEMS pressure sensor," Microsystem Technologies, vol. 16, no. 11, pp. 1921-1929, 2010.

[11] FEA Software ANSYS APDL, Version 15.0, ANSYS Element Library, http://www.ansys.com/Structures/Software. 


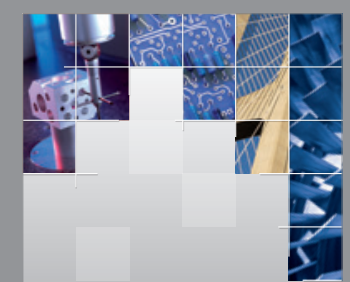

\section{Enfincering}
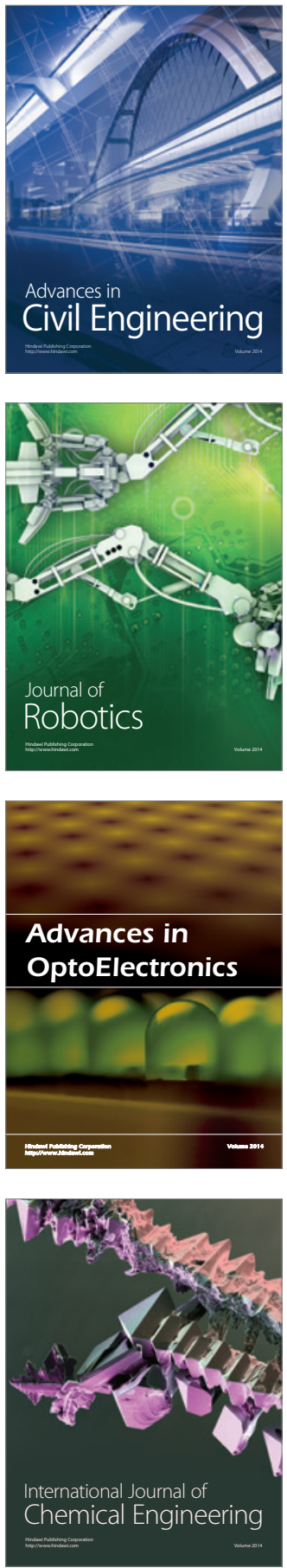

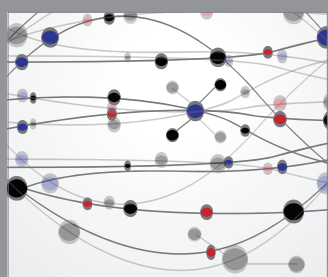

The Scientific World Journal

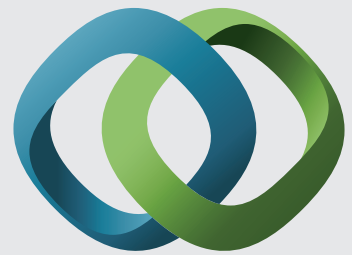

\section{Hindawi}

Submit your manuscripts at

https://www.hindawi.com
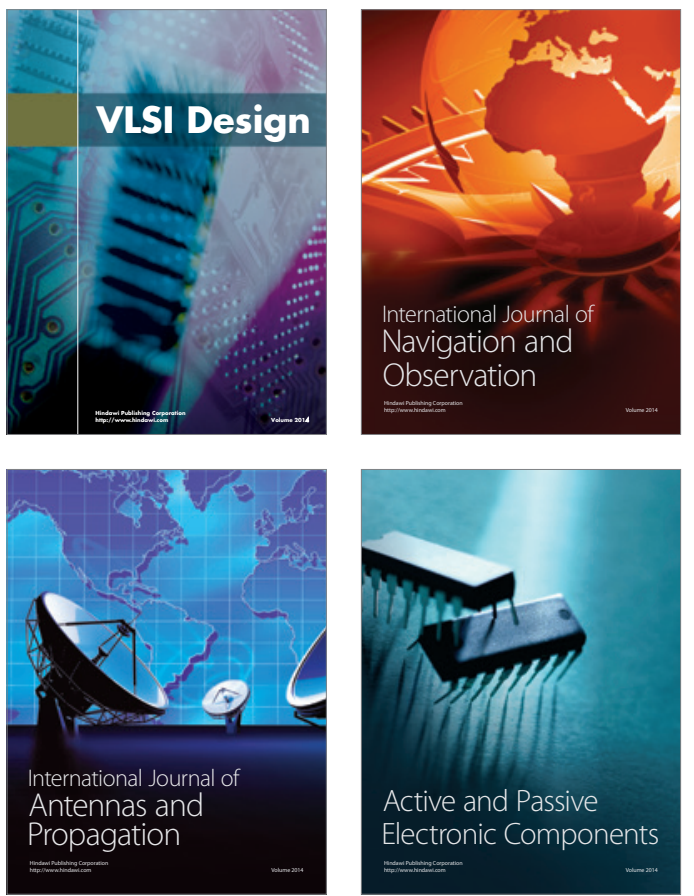
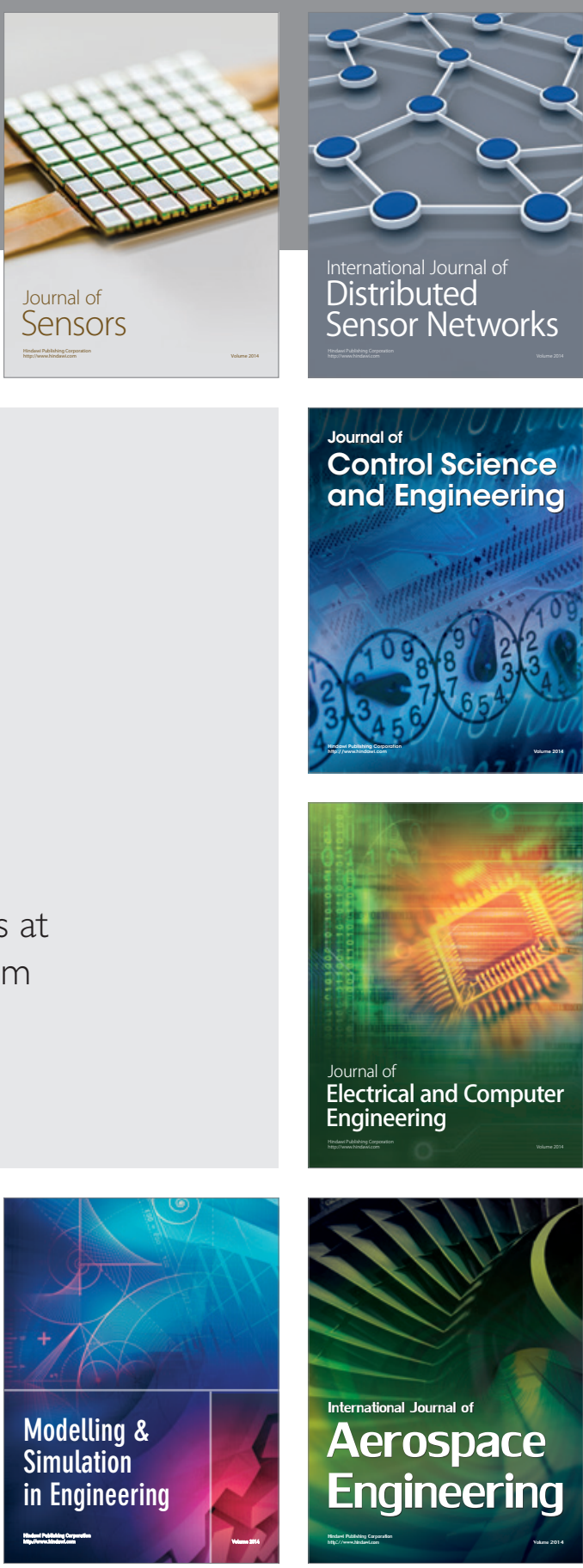

International Journal of

Distributed

Sensor Networks

$-$

Joumal of

Control Science

and Engineering
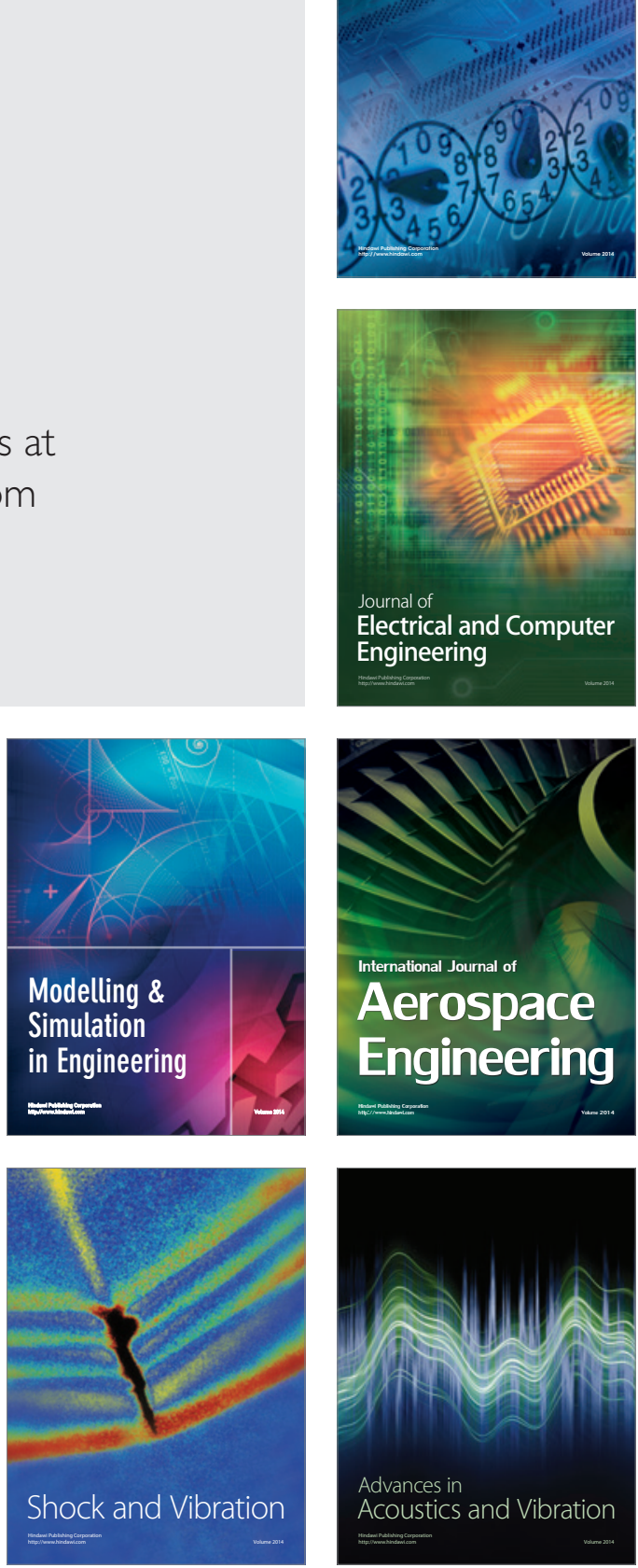